\title{
THE INFLUENCE OF THE ANGLE OF GAZE UPON THE EXCITABILITY OF THE LIGHT-SENSITIVE NEURONS OF THE POSTERIOR PARIETAL CORTEX ${ }^{1}$
}

\author{
RICHARD A. ANDERSEN ${ }^{2}$ AND VERNON B. MOUNTCASTLE ${ }^{3}$ \\ The Philip Bard Laboratories of Neurophysiology, The Johns Hopkins University School of Medicine, Department of \\ Neuroscience, Baltimore, Maryland 21205
}

Received September 8, 1982; Revised October 26, 1982; Accepted November 5, 1982

\begin{abstract}
The responses of parietal visual neurons are markedly increased during attentive fixation, as compared to those evoked in relaxed wakefulness, an effect specific for directed attention and unrelated to putative differences in the general level of arousal. Those responses are also strongly influenced by the angle of gaze, an effect observed only during directed visual attention. The change in response is smoothly graded along a meridian for about one-half the neuron population; the average spatial gradient from maximum to minimum is $78 \%$ response for a $20^{\circ}$ shift in eye position. No lateral preference was observed. For the remaining half, responses were either maximal or minimal for fixations dead ahead, and changes occurred with deviations in any direction. Angle of gaze effects were observed for neurons with foveal as well as eccentrically located receptive fields, all of which were organized in retinotopic not spatial coordinates. Control experiments showed that the effect was not produced by changes in visual background with changes in the angle of gaze, nor to changes in fixation distance, nor to variations in the intensity of stimuli viewed from different angles. The effect depends upon the position of the eye in the orbit, but is unlikely due to a direct central action of changes in nonretinal orbital afferent activity at different angles of gaze, for the effect was rarely observed with changes in the angle of gaze during relaxed wakefulness without directed visual attention. The evidence supports the interpretation that the effect is produced by a central influence of the systems controlling directed visual attention and the angle of gaze upon those linking the retinae to the parietal lobe.
\end{abstract}

We described in an earlier paper the functional properties of the visual neurons of the inferior parietal lobule, studied in waking monkeys (Motter and Mountcastle, 1981). Those properties were revealed in a behavioral state we defined as attentive fixation, which we found to facilitate the systems linking the retinae to the parietal lobe (Mountcastle et al., 1981). The responses of parietal visual neurons are more intense in this state than are those evoked by physically and retinotopically identical stimuli delivered in an alert state without attentive visual fixation. Control experiments indicated that the effect was not due to a shift in the general level of arousal between the two states compared, nor to a sensory-sensory interaction between fixation targets and test visual stimuli. We observed in the course of those experiments

\footnotetext{
' This work was supported by Grant 5 RO 1 EY03517 from the United States Public Health Service, which we gratefully acknowledge.

${ }^{2}$ Postdoctoral fellow of the United States Public Health Service, 1979-1981. Present address: The Salk Institute, San Diego, CA.

${ }^{3}$ To whom correspondence and requests for reprints should be addressed.
}

that the responses of parietal visual neurons also depended upon a second factor, the angle of gaze. We describe this effect in that which follows, together with the results of control experiments which we believe eliminate four alternative explanations of the effect: that it might be due to sensory-sensory interactions, that it might be due to differences in the depth of fixation, or to the intensity of the stimuli delivered in the comparison states, or that it is produced by a putative nonretinotopic organization of the receptive fields of parietal visual neurons. Finally, we present evidence that the controlling effect of the angle of gaze upon the visually evoked responses of parietal neurons is dependently related to the state of directed visual attention, for it is seldom observed in the comparison state of alertness without directed visual attention.

A preliminary description of these observations was presented to the Society for Neuroscience in 1980 (Andersen and Mountcastle, 1980).

\section{Materials and Methods}

The results presented here were obtained in an experimental series of 11 monkeys described in two recent 
papers that contain descriptions of the behavioral and electrophysiological methods used (Motter and Mountcastle, 1981; Mountcastle et al., 1981). Briefly, adult macaque monkeys sat in a light-tight enclosure viewing from $34 \mathrm{~cm}$ distance a $127^{\circ} \times 150^{\circ}$ translucent tangent screen. Trained animals indicated their capture of a $0.3^{\circ}$ target light by closure of a signal key. They were required to maintain fixation of the light and indicate their detection of its dimming by releasing the signal key, for liquid reward. The fixation targets were generated by a laser beam back-projected upon the screen and positioned by movable galvanometers, under computer control. Luminous test stimuli were also back-projected upon the screen. They were $6^{\circ} \times 6^{\circ}$ squares, ranged in intensity from 0.2 to $2.0 \log$ units above a uniform 1 to $2 \mathrm{Cd} / \mathrm{m}^{2}$ background, and could be positioned anywhere on the screen, turned on and off, or moved in any direction at velocities up to $600^{\circ} / \mathrm{sec}$, under program control. Electroculograms (EOGs) were recorded through $\mathrm{Ag}-\mathrm{AgCl}$ electrodes implanted in the orbital rims. The EOG records were linear to at least $20^{\circ}$ deviations from straight ahead in any one of the four cardinal dircctions, with coefficients of variation of 2 to $5 \%$ at $20^{\circ}$ deviations. Analog values of EOGs were digitized at 100 to $200 / \mathrm{sec}$ and stored. If the animal made a saccade of $1^{\circ}$ or greater during a fixation period, that trial was terminated without reward and was followed by a delay of the next trial as punishment.

The electrical signs of the impulse discharges of single cortical neurons were recorded from extracellular positions with Pt-Ir, glass-coated electrodes passed through the intact dura into the parietal cortex. Usually one microelectrode penetration was made during each day's recording session of 6 to $7 \mathrm{hr}$. The cranial chamber was then closed and the animal returned to its living cage overnight. Recording continued for 2 to 4 weeks in each hemisphere. The time intervals between impulses were digitized at $10,000 / \mathrm{sec}$ and the values stored. Conventional spike train analyses were used off-line for studies of the time relations between neuronal discharges, behavioral events, and eye positions and movement. All of the neurons considered here were positively identified as light sensitive by their responses to projected visual stimuli. The locations of microelectrode penetrations of this series are given in part in Figure 2 of Motter and Mountcastle (1981); all passed into area 7-a of the inferior parietal lobule.

The animal's head was fixed with its coronal plane parallel to the tangent screen. The intersection of the central line of gaze with the screen was taken as the zero point of a screen coordinate system of visual angle. The responsiveness of light-sensitive neurons was tested at different visual angles by placing the fixation target at different locations on the screen and presenting either stationary or moving test stimuli at equal distances and directions from those fixation points; an example is given in the upper panel of Figure 1. Thus retinotopically identical zones were tested at different angles of gaze.

Definitions of behavioral states. The responses of parietal visual neurons were examined at different angles of gaze and in two behavioral states. The first, called the task state, is that described above. We have presented evidence that this state of maintained fixation coupled with a high probability of correct detection is one of directed or focused visual attention (Mountcastle et al., 1981). The second is the no-task state in which the animal sat facing the tangent screen but fixated no target and executed no task; the signal key and reward tube were removed. His alertness was suggested by the EOG signs of successive eye pauses and brisk saccadic eye movements and by remote television monitoring. Test visual stimuli identical to those delivered in the task state were presented at nine locations on the tangent screen in a randomized spatial and temporal sequence. Eye position and stimulus position data were collected along with the interspike interval sequences and used with off-line analysis programs to reconstruct the approximate retinotopic positions of stimuli. A series of control experiments indicated that the major variable between the two states is the presence of directed visual attention in the first and its absence in the second (Mountcastle et al., 1981).

Comparison of angle of gaze effects in the task and no-task states. The angle of gaze effect was examined in the no-task state by comparing the responses evoked during eye pauses made at different gaze angles, in which stimuli were delivered at similar retinotopic locations. Trials from the no-task state were chosen for analysis if the animal made no saccade larger than $2^{\circ}$, nor any series of smaller saccades summing to $5^{\circ}$ during a 500 to 800 msec period centered around a 100 - to $300-\mathrm{msec}$ stimulus. For example, the upper plot of Figure 7 shows the fixation positions during eye pauses which met the stability requirements, during an experimental run in which 63 of 91 trials met these criteria. The dotted lines in Figure 7 define three fields of fixation; two of these, 1 and 2 , are used for the stimulus position plots of the two middle graphs of Figure 7 for which vector subtractions of eye position during fixation were made for each point. Thus the origins of these plots represent the transformed fixation points and the crosses the retinotopic positions of the stimuli delivered. The plot to the right gives the retinotopic loci of stimuli delivered during fixation positions between dead ahead and $15^{\circ}$ to the left; that on the left for fixations greater than $15^{\circ}$ left. The small boxes outlined with dots in each graph enclose the retinotopic positions of the testing stimuli for the two fixation areas. The outer box indicated by heavy dashed lines is the receptive field of the neuron, which extended to at least $50^{\circ}$ in all directions from the central line of gaze. Standard statistical methods were used to test the significance of responses to stimuli compared to prestimulus levels of activity and whether significant differences existed between responses evoked at different angles of gaze. The histograms shown are unsmoothed.

\section{Results}

\section{The data base}

The data analyzed and the results described below were obtained in an experimental series in which 564 light-sensitive neurons were identified and 418 studied under' conditions in which behavioral state and the stimuli delivered were controlled. The response properties of those cells and their sensitive regulation by the behavioral state of directed attention were described in earlier 


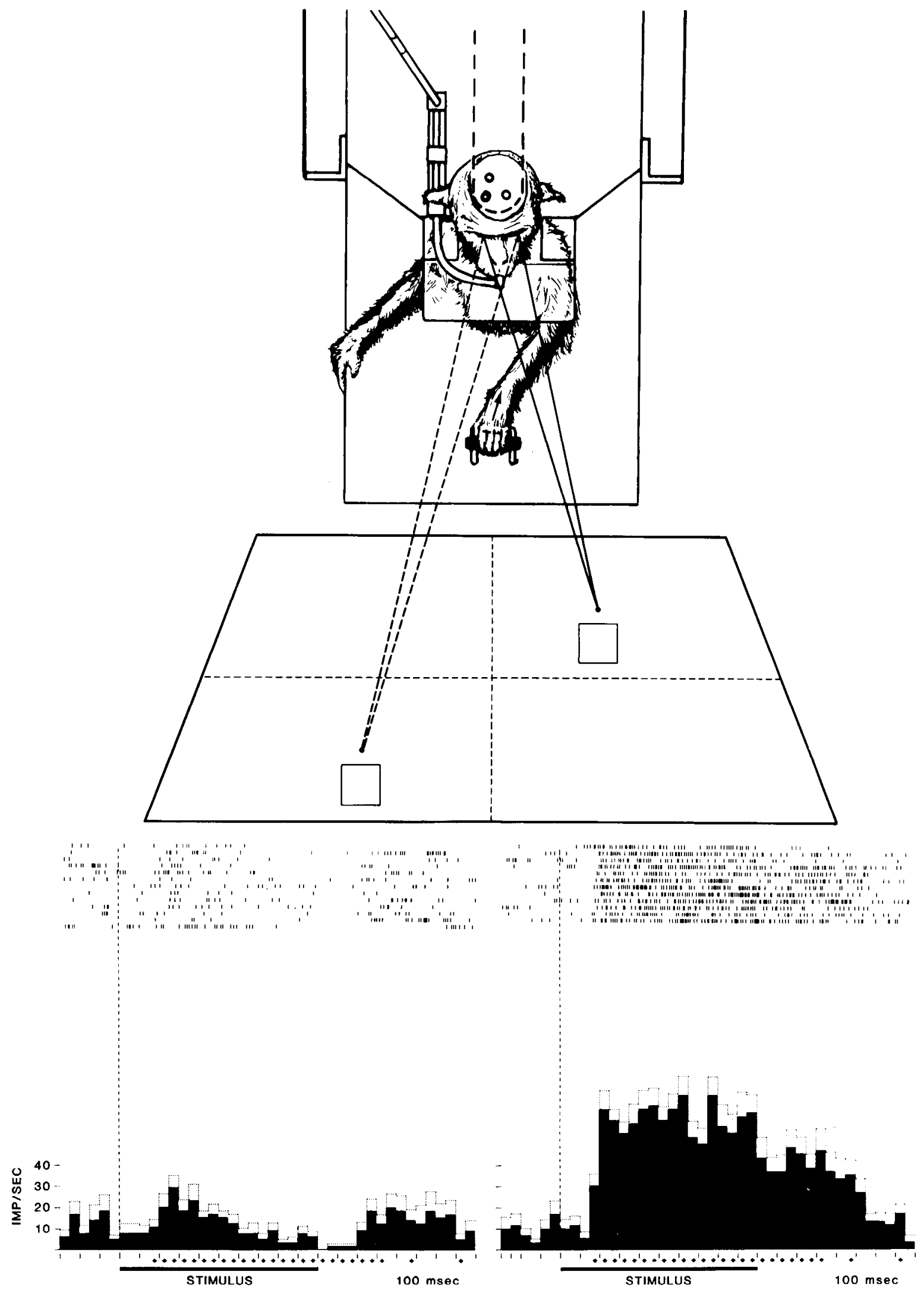

Figure 1. Illustration of the experimental arrangement and the effect of the angle of gaze upon the responses of a parietal visual neuron to light stimuli. The animal fixated a small target light placed at a series of positions on the tangent screen, with the head fixed. The results obtained at two positions are shown here. At each fixation position a $6^{\circ} \times 6^{\circ}$ square test stimulus of 1 -sec duration was flashed $10^{\circ}$ above the fixation point. The tests at different gaze angles were presented in the same experimental run in a randomized spatial and temporal order. Impulse replicas are shown below the screen; each line represents a single trial, each short upstroke the discharge of an impulse by the neuron. The impulse replicas and summing histogram to the right show the responses to retinotopically similar stimuli delivered during fixations left $20^{\circ}$, down $20^{\circ}$; those to the left for fixations right $20^{\circ}$, up $20^{\circ}$. The dotted lines above the bins of these and all other histograms in the figures that follow indicate the standard errors 
papers (Motter and Mountcastle, 1981; Mountcastle et al., 1981). We have analyzed the responses of 102 of those neurons to retinotopically and physically identical light stimuli delivered as the animal gazed in different directions. Fifty-four were studied as the animal fixated a target light located at different positions on the tangent screen, in the state of focused attention we define as the task state. Thirty were studied with stimuli delivered during eye pauses at different angles of gaze as the animal sat gazing here and there upon the tangent screen, in a state of alertness without directed attention we define as the no-task state. Eighteen additional cells were studied for angle of gaze effects in both the task and no-task states. The remaining light-sensitive neurons studied did not enter this population for analysis because angle of gaze studies were not done or, if initiated, were not completed. The angle of gaze effects we describe below have been observed for parietal visual neurons with foveal and those with nonfoveal receptive fields (Motter and Mountcastle, 1981) and in each of the cellular layers of the cortex. The population of cells studied for this effect is too small to permit an analysis of their laminar or columnar distribution.

\section{The responses of parietal light-sensitive neurons to visual stimuli are influenced by the angle of gaze during attentive fixation}

The phenomenon is illustrated in Figure 1, in which the monkey and the tangent screen are shown as if seen from above. The small fixation light came on at one of nine locations on the screen (only two are shown in Figure 1) for each trial of the run. During each of those trials, a $6^{\circ} \times 6^{\circ}$ test light centered $10^{\circ}$ above the fixation point for that trial was flashed for 1 sec; trials at different locations were sequenced in a randomized spatial and temporal order. The impulse replicas and impulse density histograms to the right of the figure show the intense responses evoked during fixation into the left lower quadrant of the visual field, at coordinates $-20^{\circ},-20^{\circ}$. They contrast with the greatly reduced responses evoked as the animal fixated into the right upper quadrant of the visual field $\left(+20^{\circ},+20^{\circ}\right)$, shown to the left in the figure. The two histograms of Figure 1 show that deviation of the angle of gaze affects both the intensity and the temporal pattern of visually evoked responses. The intensity changes are regular and consistent; those in temporal pattern are more capricious, and we have not studied them in detail. The results of the full field study for the neuron of Figure 1 are shown in Figure 2. There it can be seen that the facilitatory effect of deviations of the angle of gaze was a gradual one, reaching its maximum with fixalion positions in the lower half of the field and especially in the left lower quadrant. Some of the relations observed are illustrated for five additional neu- rons in Figure 3; for each of these, $20^{\circ}$ deviations (or greater) of gaze in one direction from the central line of gaze produced a clear facilitation of the responses to retinotopically similar stimuli.

We observed a variety of spatial distributions of the effect of the angle of gaze upon the responses of parietal light-sensitive neurons to visual stimuli. For nearly onehalf of the cells studied the effect was distributed smoothly across the field of gaze, at least as measured along a single meridian. An example is shown in Figure 4. This neuron was most sensitive to upwardly moving stimuli. In the experiment illustrated, excitability was tested at a series of fixation points located at $10^{\circ}$ intervals along the vertical meridian. The stimulus in each case was a $6^{\circ} \times 6^{\circ}$ square light moving upwardly at $25^{\circ} / \mathrm{sec}$, with its $25^{\circ}$ path centered upon the point of fixation. There is a smooth gradation of the effect from below upward to a maximum for fixations at $20^{\circ}$ above the central line of gaze.

The four graphs of Figure 5 illustrate the types of spatial distributions observed. Those shown in the upper two graphs resemble that of Figure 4. Their normalized mean rates of discharge change smoothly with deviations of gaze along a single meridian, either up or down or to the contralateral or the ipsilateral side. Each of these types has been observed in the same single hemisphere; no laterality effect has been observed in the small population we have studied. The slopes of these functions vary from about $2 \%$ to $4 \%$ change in response per degree of deviation of the line of gaze. The lower two graphs of Figure 5 illustrate cases in which deviation of gaze from the central line of vision produced quasi-symmetrical changes in response, either increases (lower left) or decreases (lower right) as compared to the response evoked during fixation dead ahead.

We tested 72 parietal visual neurons in the manner illustrated in Figures 1 to 4 . Forty-four of these $(61 \%)$ showed significant (criterion $p=<0.05$ ) and frequently strong changes in response to visual stimuli with changes in the angle of gaze. Moreover, many of the 28 cells classed as having no change were tested at only $10^{\circ}$ deviation of gaze and some only along a single meridian. More complete testing would almost certainly have increased the percentage of parietal visual neurons with angle of gaze effects.

The magnitude of the angle of gaze effect for each neuron was given a numerical value as the ratio of the two mean responses evoked by retinotopically similar stimuli at two angles of gaze separated by $20^{\circ}$. These $20^{\circ}$ segments were generally chosen from the steepest part of the response/angle of gaze functions (see Figure 5). The median ratio for 38 neurons was 3.8. That is, for the steepest downward $20^{\circ}$ segment of the response/angle of gaze functions, the typical neural discharge in response

of the means for the bins. Diamonds below corresponding bin pairs of the two histograms indicate that a significant difference exists between the means of those bins $(p=<0.05)$. Time marks on abscissae $=100$ msec; divisions on ordinates $=10$ impulses/ sec. The significance of a response to a visual stimulus was judged for each gaze angle (paired t test, $p=<0.05$ ). The responses at different gaze angles were compared by subtraction of the rate of discharge in the prestimulus period from that in the poststimulus period for each trial, and the populations of remainders tested for significance $(p=<0.05)$. These net responses were also used to form the ratios of Figure 6 and the normalized numbers for the graphs of Figure 5. 

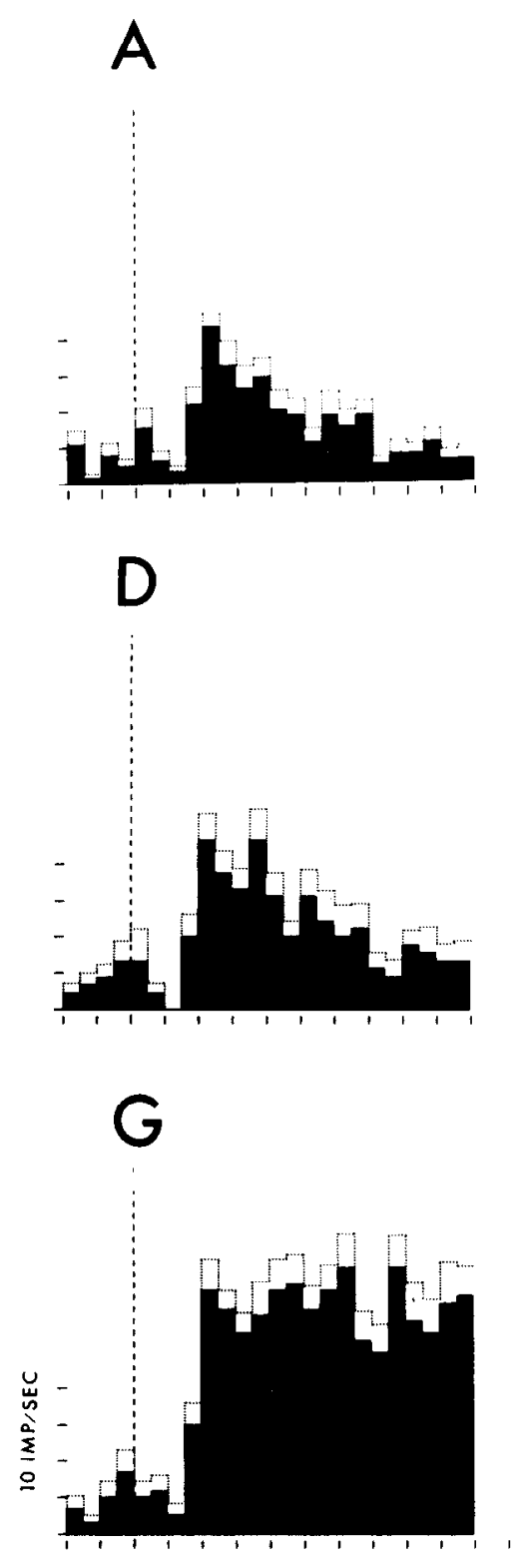

100 MSEC
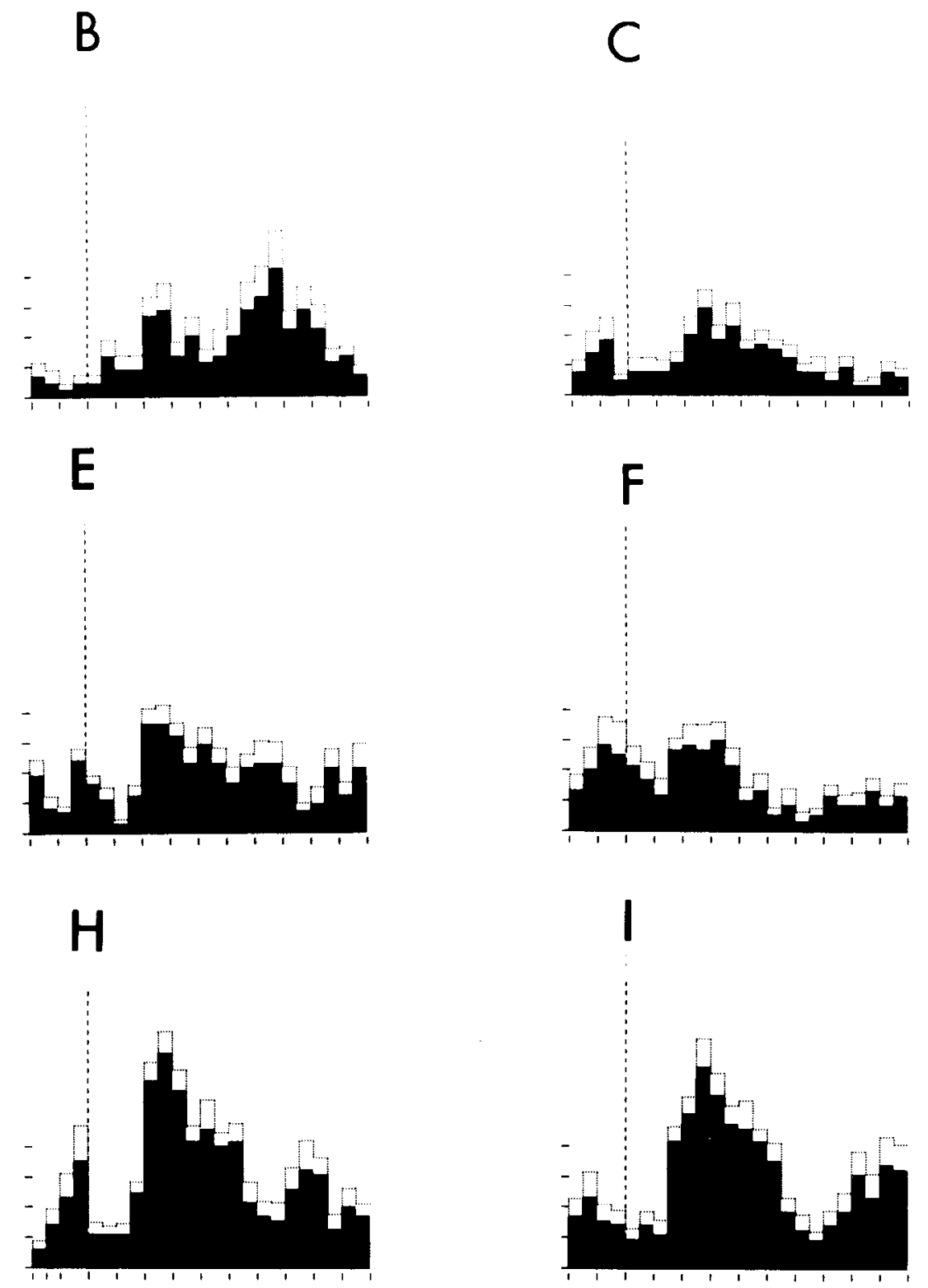

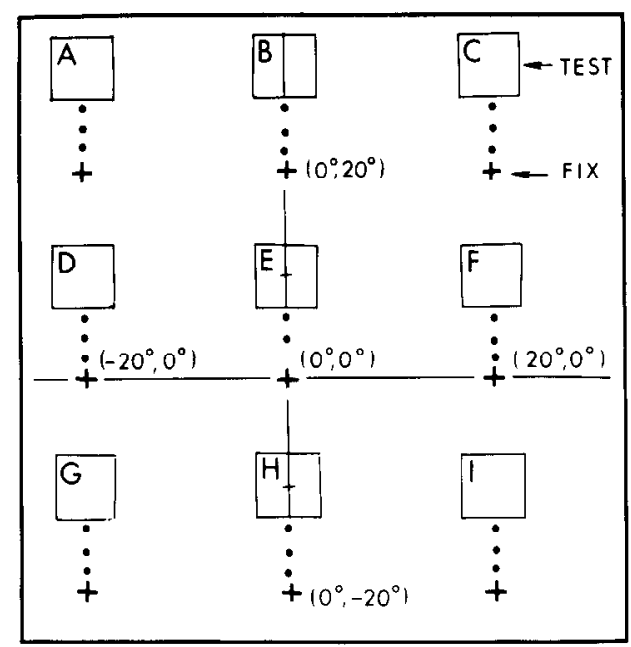

Figure 2. The results of the full field study for the neuron of Figure 1. Tests were made with fixations at each of nine directions of gaze; in each case the test visual stimulus was delivered $10^{\circ}$ above the point of fixation, as shown by the inset below. Stimuli began at the dotted vertical lines and lasted for $1 \mathrm{sec}$. All trials were arranged in a randomized spatial and temporal sequence, and the results were later pooled by classes. Histogram scales and conventions are as for Figure 1. The map shows the gradual trend toward increased responses with deviations of gaze toward the left and lower parts of the field of gaze. 

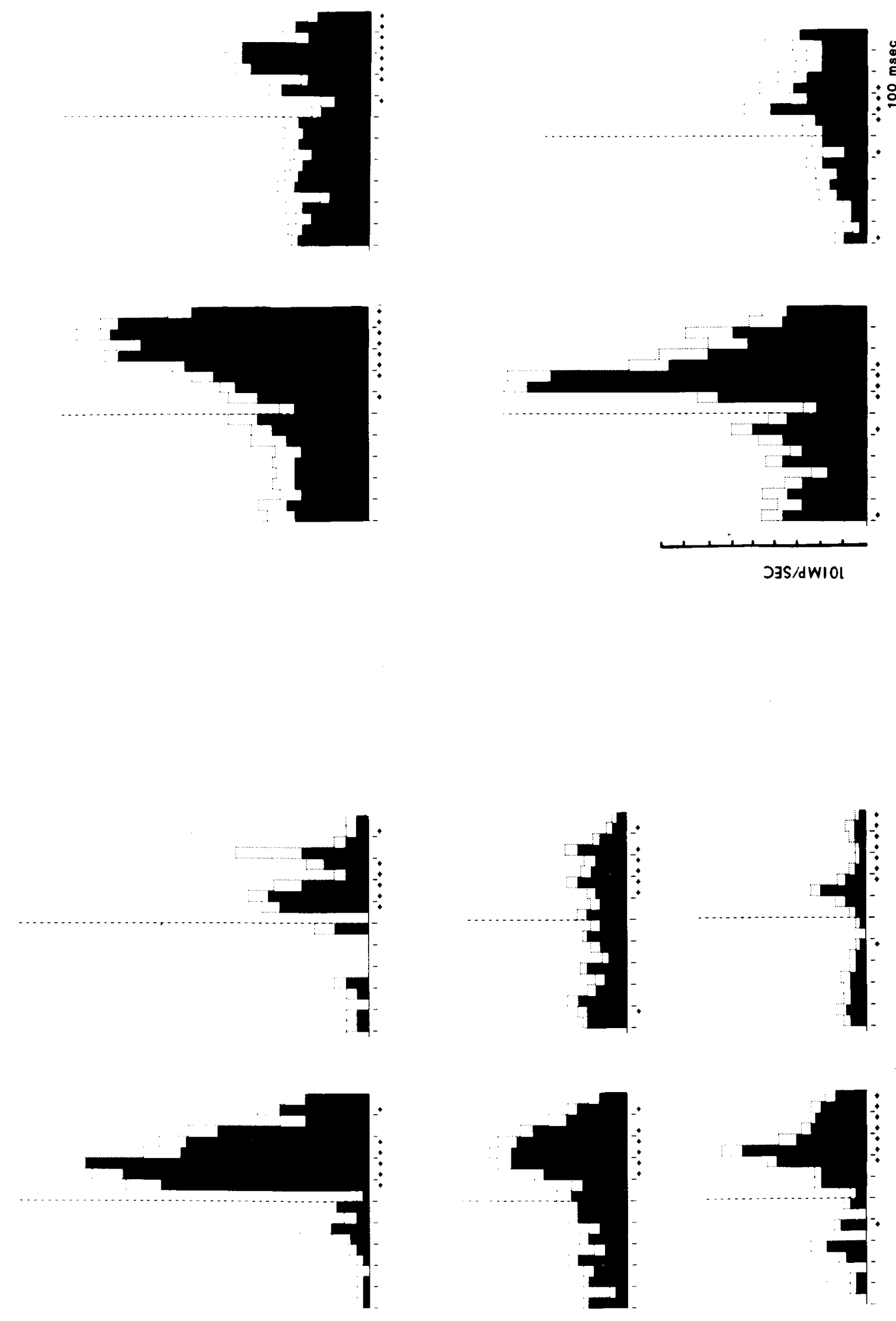

๑ $\Xi \cong . \Xi$

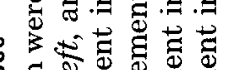

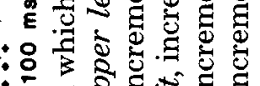
$=5$.

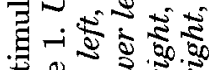
का

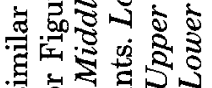

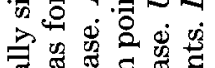

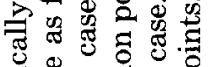

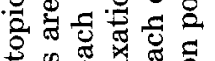

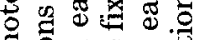
$\Xi$. 过造递 8 讨 ह 영

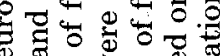

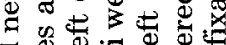

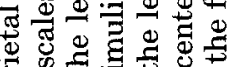

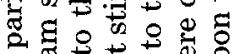

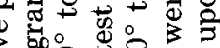

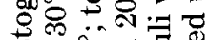

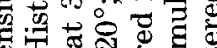

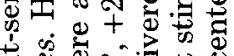

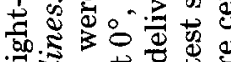
$=$ 马

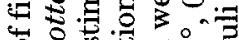

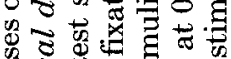

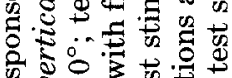

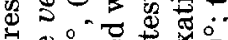

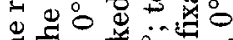
$5 \pm$ का 范 击

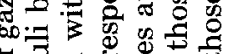

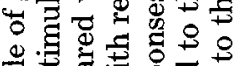

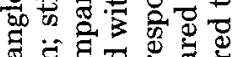

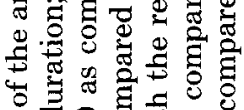

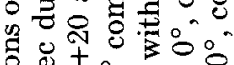
o ๘ के की 0

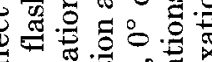
क

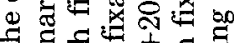

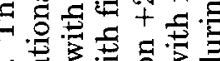

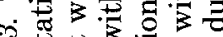

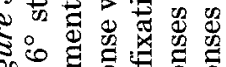

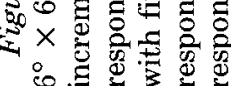




\section{FIXATE}
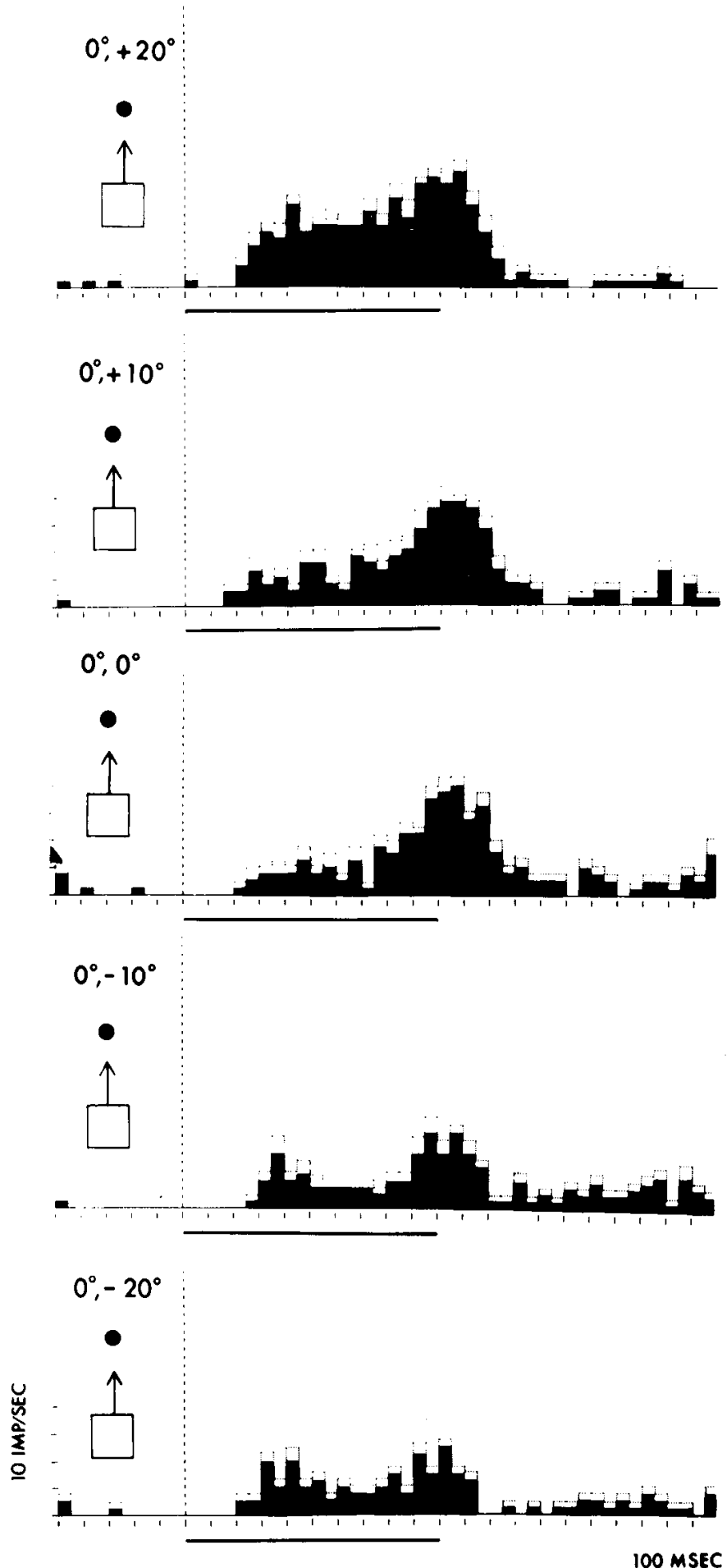

Figure 4. Illustration of the effect of angle of gaze upon the responses of a parietal visual neuron; here the effect is smoothly graded along the vertical meridian. Fixation positions in each case are shown by insets to the left; in each the stimulus was a $6^{\circ} \times 6^{\circ}$ square light moving upward at $25^{\circ} / \mathrm{sec}$, with its $25^{\circ}$ traverse centered upon the fixation point. Horizontal lines indicate periods of stimulation. Histogram scales and conventions are as for Figure 1. Trials from all classes were delivered in a randomized spatial and temporal order. to visual stimuli decreased by $73 \%$, or $3.65 \%$ per degree. The distribution of these ratios is given in Figure 6.

The interaction of the influences of the angle of gaze and the state of attentive fixation upon the excitability of parietal visual neurons

The evidence presented above indicates that the responses of many light-sensitive parietal neurons to visual stimuli are conditional in nature. The intensity and, indeed for many cells, even the presence of a response depends upon both the retinotopic location of the visual stimulus and its spatial position, i.e., the angle of gaze between the established central line of gaze and the direction of gaze at the time of stimulation. This spatial effect is a powerful one, as shown by the records and histograms of Figures 1 to 4 . We have previously observed that the excitability of parietal visual neurons is also controlled by the state of directed or focused attention (Mountcastle et al., 1981). Briefly, the responses of parietal light-sensitive neurons to visual stimuli are facilitated during the state of attentive fixation of a target or of an untargeted locus in space (the task state), as compared to their responses to physically and retinotopically identical test stimuli delivered during the eye pauses of alert wakefulness (the no-task state). Control experiments showed that the response increment during attentive fixation is not due to a shift in arousal between the two states compared, nor the result of a sensory-sensory interaction between target and test stimuli. We sought to discover whether any dependence or interaction exists between these two control states (i.e., attentive fixation and its absence) and the angle of gaze.

We examined the effect of the angle of gaze upon the responses of parietal visual neurons to light stimuli in the task and no-task states by comparing those evoked during eye pauses in the no-task state with those evoked by identical stimuli delivered in the task state, as the animal fixated a target light and performed the dimming detection task. We collected a large number of trials in the notask state, including those at many eye deviations, and an equally large number at different fixation points in the task state with constant retinotopic positions of the test stimuli. This allowed us to determine if the angle of gaze at the instant of stimulation influenced the responses in the absence of the directed visual attention of the task state.

The effects of the angle of gaze upon the responses of parietal visual neurons in the state of attentive fixation were described in earlier sections. By contrast, of 48 neurons tested in the no-task state, only five showed a significant effect of gaze angle upon the responses to visual stimuli, with a median ratio of 2.9 between maximal and minimal responses. The rarity of the effect in the absence of attentive fixation persisted when we tested for the effects of eye deviations along several meridians. Of the 48 neurons referred to above, 18 were tested for the angle of gaze effect upon visual responses in both the task and the no-task states. Of these, nine showed angle of gaze effects in the task state, but no single neuron did so in the no-task state.

Figure 7 illustrates the common finding, that the angle of gaze has no effect upon the responses of parietal visual 

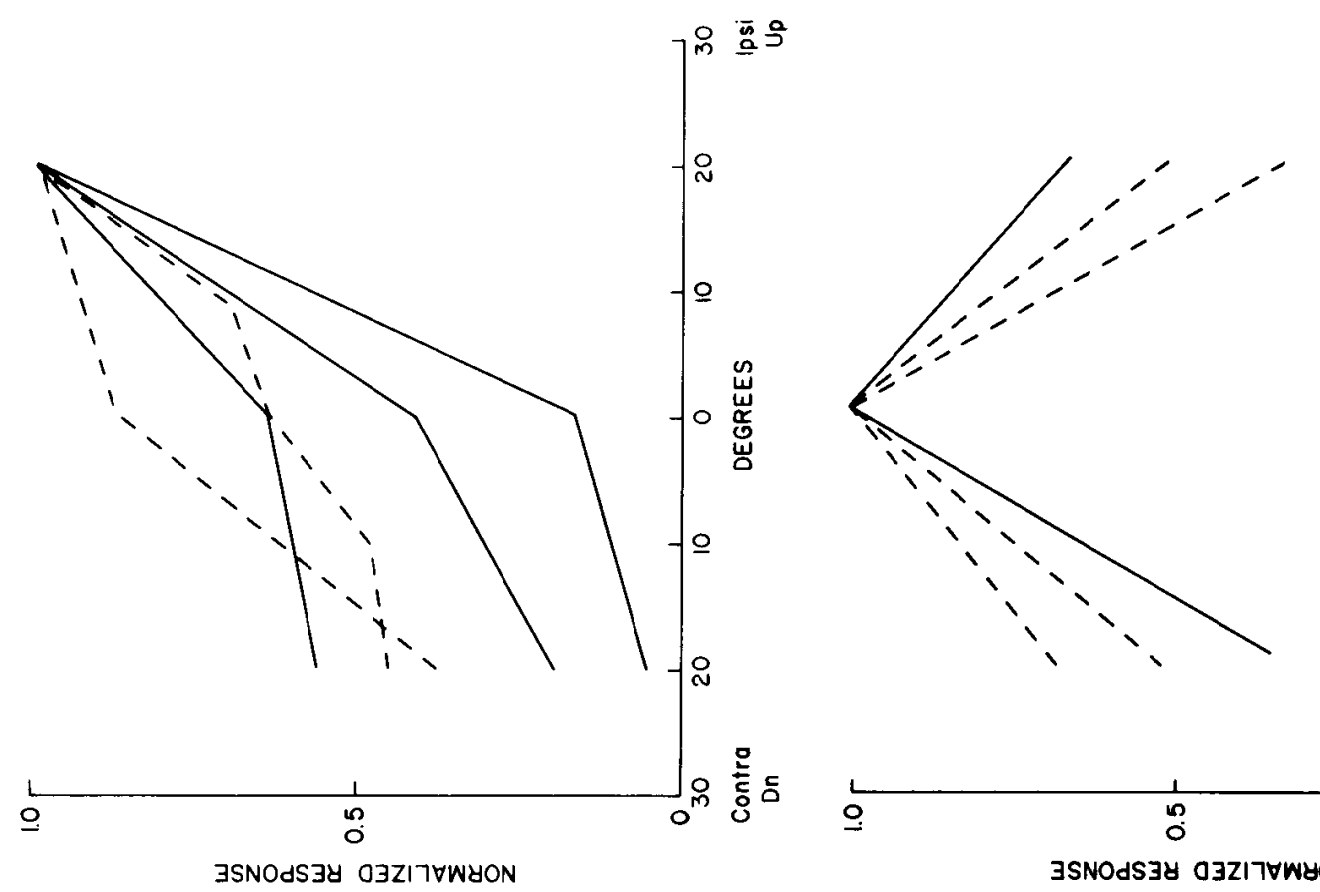

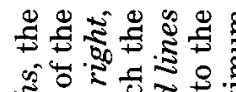

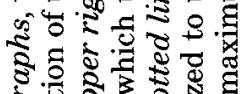

o.

政

要起

疍

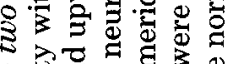

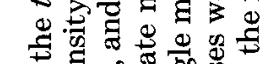

ก $\quad$ के

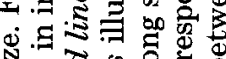

चั

ब 8 क्ष

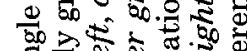

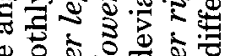

स

प

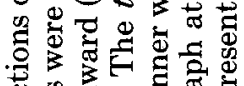

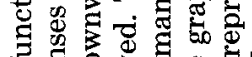

๘

क क

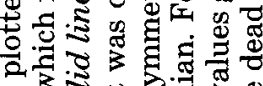

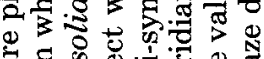

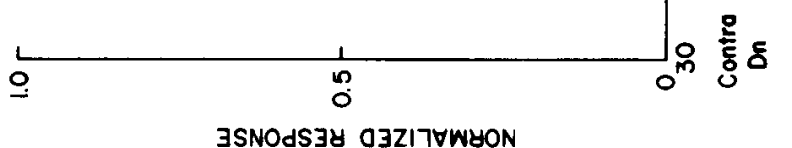

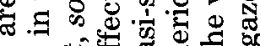

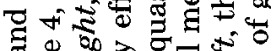

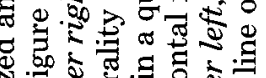

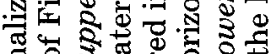
论

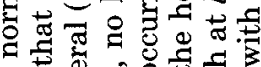

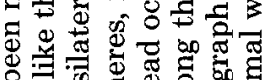

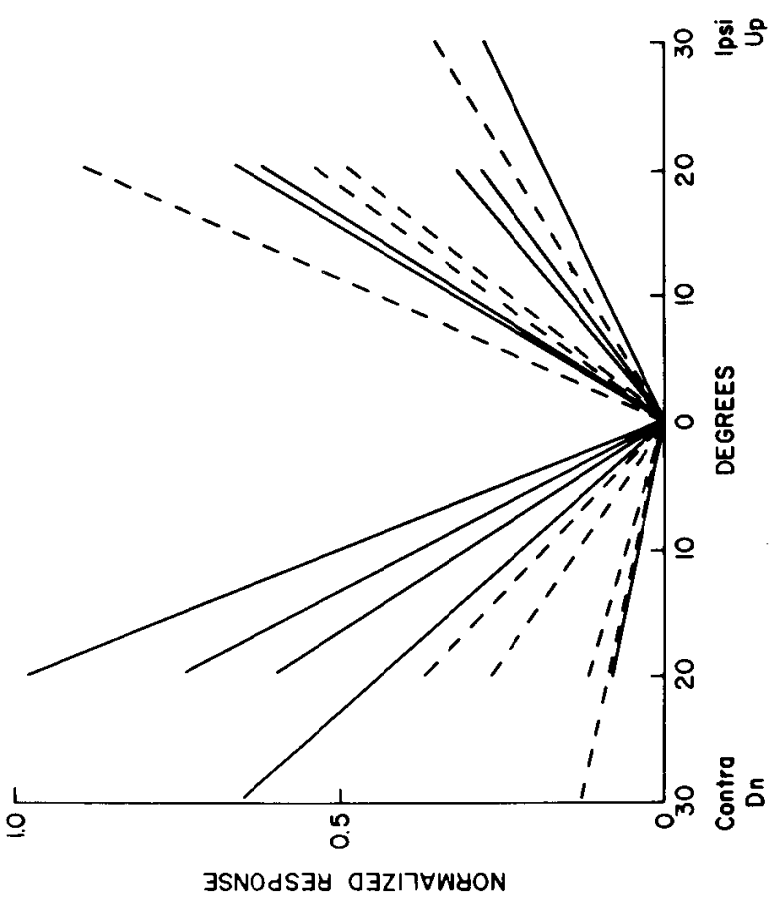

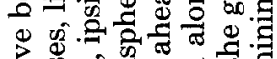

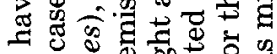

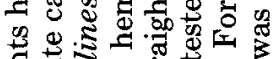

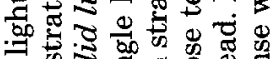

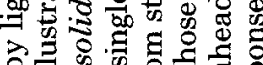

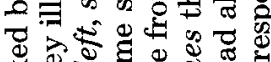

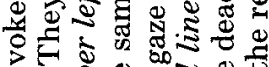
更

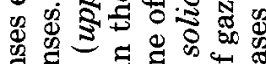

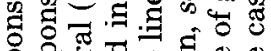

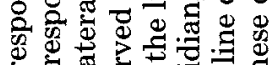
छ

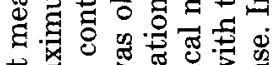

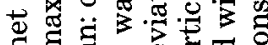
व

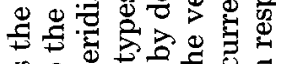

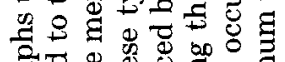

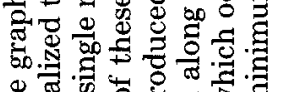
का 政

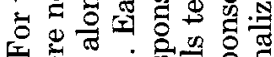

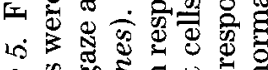

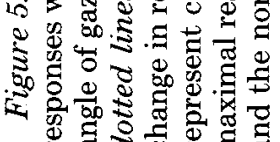




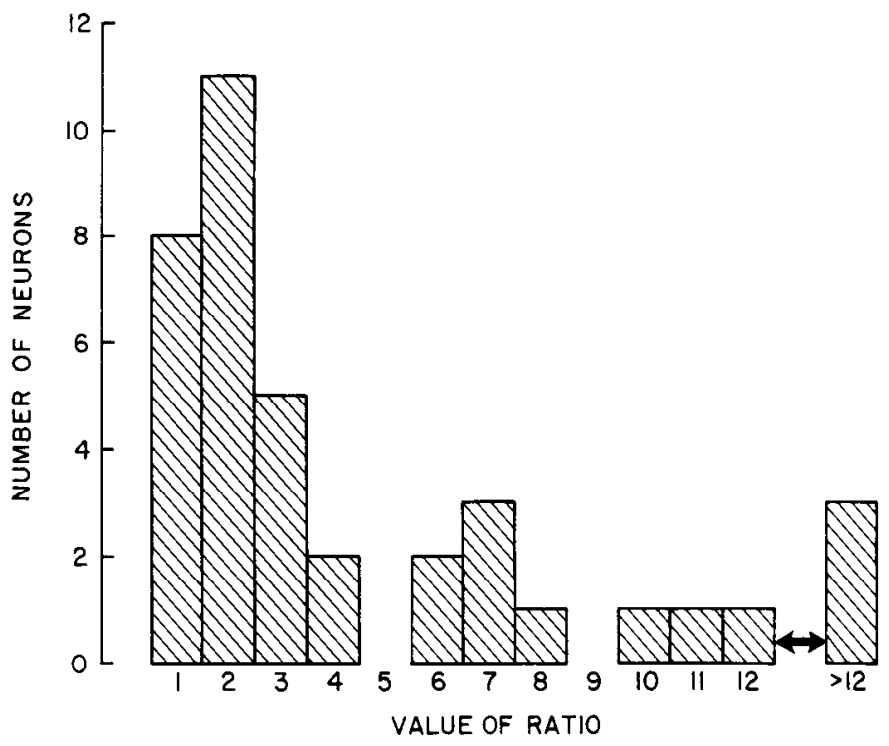

Figure 6. Distribution of the ratios between the responses evoked by retinotopically similar stimuli at two angles of gaze, in each case separated by at least $20^{\circ}$. If more than two $20^{\circ}$ segments were tested for a single neuron, the ratio entered is that for the segment with the larger angle of gaze effect. In the few instances in which the angle tested was $30^{\circ}$, the ratio was multiplied by two-thirds. $N=38$; median value of the ratio $=$ 3.8 .

neurons, in the absence of directed visual attention. This neuron was chosen for illustration because it did respond in the no-task state, although those responses were much reduced from those evoked in the task state. This allowed an examination of the influence of the angle of gaze in the two behavioral states. For the analysis of Figure 7, eye pauses in different sectors of the field of gaze were classed together, as shown by the plot of pause positions in the top graph of Figure 7. The areas outlined by the heavy dashes in the two middle graphs comprise the spatial extent of the receptive field of this cell; it extended in all direclions beyond the limits of our test area. The crosses of the middle chart to the left indicate the retinotopic positions of stimuli delivered during eye pauses with gaze directed to the left of the $15^{\circ}$ vertical line, zone 1 of the upper chart. The crosses of the middle chart to the right indicate the retinotopic positions of stimuli delivered during eye pauses directed between dead ahead and that $15^{\circ}$ left vertical, zone 2 of the upper graph. The stimulus positions enclosed in each of the dashed line boxes of the middle graphs were chosen for comparison, for they cover virtually identical retinotopic zones. The impulse replicas and impulse density histograms below show no significant differences in the responses evoked by the two sets of stimuli delivered at identical retinotopic positions, but at different angles of gaze. Figure 8 gives the results obtained in a study of one of the five neurons for which we did observe an angle of gaze effect upon the responses of parietal visual neurons in the no-task state. The experiment and the analysis resemble those described for Figure 7, except that the zones chosen for analysis were determined by horizontal lines, as shown in the upper graph. We conclude on the basis of these observations that for the large majority of parietal visual neurons the powerful effect of the angle of gaze upon the excitability of systems linking the retinae to the parietal cortex is dependently related to another state control, that of directed visual attention. The rarity of angle of gaze effects in the no-task state is not due to a uniform unresponsiveness of parietal light-sensitive neurons in that state, as compared to that of attentive fixation. Thirty-two of the neurons in our test population did show significant responses to visual stimuli in the notask state, even though they were much reduced from the responses evoked in the task state. Of these 32 , only the five described above revealed any effect of the angle of gaze upon their (reduced) responses.

We described in a recent paper a difference in the excitability of parietal visual neurons between the state of attentive fixation and the no-task state described above (Mountcastle et al., 1981). We have now analyzed again the data for 48 of the 55 neurons which formed the base of the first portion of that study to determine whether an influence of the angle of gaze in the no-task state could have contributed to the differences observed. We found that for no single neuron could an observed reduction in response in the no-task state be accounted for by an angle of gaze effect. An illustration of this result is given in Figure 9. Vigorous responses were evoked in the task mode by stationary stimuli delivered $20^{\circ}$ below the fixation point. The responses to identical stimuli delivered in the no-task state are reduced; this reduction is shown by the histograms of the left panel. For the histograms of the center and right panels of Figure 9, the responses evoked in the no-task state, grouped together for the left panel, are separated into six populations which differ from each other by differences in the angle of gaze. There are no significant differences between these sets of responses. Thus the reduction in light sensitivity illustrated to the left-the effect of the absence of directed visual attention-cannot be accounted for by an angle of gaze effect operating in the no-task state. The reduction in response associated with the notask state is independent of the angle of gaze at the time of stimulus presentation. This lends further support, we believe, to our conclusion that the angle of gaze effect is dependently related to the state of attentive fixation.

\section{Control experiments}

Background interaction. One alternative explanation of the effect of changes in the angle of gaze upon the responses of parietal visual neurons is that even slight differences in the visual backgrounds in the two comparison cases might interact differently with the test stimuli to produce the effects observed. We have controlled for this possibility by comparing the effects of changes in gaze angle on visually evoked responses in light and darkness. A typical result is illustrated in Figure 10. The histogram at upper left sums responses evoked by stimuli moving upward through a fixation point $30^{\circ}$ to the left along the horizontal meridian. This is to be compared with the responses evoked by retinotopically similar stimuli delivered during fixations dead ahead, summed by the histogram at upper right. The strong effect of this shift in the angle of gaze was virtually the same when the 
experiment was repeated in darkness, as shown by the two histograms below. In each of these experiments, trials for fixations at the two positions were sequenced in a pseudorandom order. We observed similar results for each of the 12 neurons tested in the manner illustrated in Figure 10; i.e., even though some changes in the overall magnitude of responses did occur upon shift from a lighted to a dark environment, the differences for the two angles of gaze remained. We conclude that the angle of gaze effect is not produced by differences in sensorysensory interaction between stimuli and background.

Controls for the differences in fixation distance and stimulus intensity. The use of a tangent screen for the display of fixation targets and test stimuli introduces three other variables which could contribute to the angle of gaze effects we observed. First, different deviations of the fixation target from the center of the screen elicit different fixation distances, for a close focus is required for detection of the slight dimming of the fixation light in our experimental arrangement. Second, deviations of the test stimulus from the locus of the fixation target move it out of focus. Moreover, deviations of the test stimulus reduce its intensity, and the reduction of intensity increases with greater deviations from the center of the screen. This is so because our tangent screen allows maximal trans-illuminance when viewed at an angle normal to its surface but reduces the intensity of projected images for all other angles of viewing. We have controlled for the effects of these variables by comparing the angle of gaze effects upon responses evoked by stimuli placed at positions symmetrical with respect to the center of the screen, and the central line of gaze, and at locations which required identical fixation distances of the target and of the testing stimuli. An example is given in Figure 11. In this case three test stimuli located $20^{\circ}$ apart along the vertical meridian were presented in three different spatial positions: with the middle stimulus located $20^{\circ}$ above, at the center of screen coordinates, of $20^{\circ}$ below that center, as shown by the diagrams to the left in Figure 11. In each of the three cases, the fixation target was presented in the center of the middle test stimulus, marked $X$ in the diagrams to the left. This test arrangement was possible because this neuron was related to a large receptive field centered upon the region of the fovea. Thus the depths of fixation required, as well as the intensities of the testing stimuli labeled $B$ in the upper and lower trios of Figure 11, were identical. The replicas of the responses evoked by those stimuli (labeled $B$ in the upper and lower sets of replicas to the right in Figure 11) show a marked angle of gaze effect. In this case the effect was much greater for upward deviations than for downward ones. We have repeated this experiment for a number of parietal visual neurons with uniformly similar results. We conclude that the differences in the responses evoked by retinotopically identical stimuli, delivered at different angles of gaze, are not due to differences in stimulus intensity or to changes in depth of fixation or relative depth of fixation point and testing stimuli, but to the difference in the angle of gaze itself.

The question of the coordinates of organization of receptive fields. It is obvious that if the receptive fields of parietal visual neurons were organized in a spatial coordinate system, and thus did not move with changes in the line of gaze, the results we have described could be attributed to a shift of the testing stimulus into or out of the nonretinotopic receptive field of the cell under study, with changes in gaze angle. We have tested for this possibility in all of our studies of parietal visual neurons and have not observed a clearly established case of a nonretinotopic receptive field. The problem is a difficult one experimentally, for the receptive fields of parietal visual neurons are so large that many extend beyond the edges of our $100^{\circ} \times 100^{\circ}$ test area on the tangent screen. Moreover, the outer edges of these large receptive fields can only infrequently be brought onto the test area by lateral deviation of the eyes; i.e., the receptive fields extend to the far lateral (or upper or lower) edges of the visual fields. We have been able to test for retinotopy in 25 of the 44 neurons of this study for which a significant effect of change in the angle of gaze upon visually evoked responses was demonstrated. Such a test required, of course, that the angle of gaze effect not be absolute, i.c., that there be responses and fields to examine at each of the angles of gaze. We could establish the inner and outer borders of the receptive fields for six of these cells, using stationary stimuli. For the 19 other neurons we could establish only the inner border (i.e., the foveal side) of the receptive fields, four with stationary and 15 with moving stimuli. We tested for movement of the border(s) of the receptive fields with changes in gaze angle in each of these 25 cases. The result was clear: in each case the receptive field border did move an appropriate distance with changes in the angle of gaze. The only change we noted, for a few cells, was a symmetrical expansion of the receptive field when deviation of the line of gaze produced a strong facilitation of response. In addition, for all the neurons examined that showed no effect upon response caused by changes in the angle of gaze, we observed the appropriate shift in the receptive field border(s) with shifts in gaze angle.

We conclude that the receptive fields of the population of neurons we have studied are uniformly retinotopic in nature and that the angle of gaze effect is not produced by the presence of nonretinotopic receptive fields. Our results suggest that there is a conjunctive interaction between the "spatial" fields mapped by changes in the angle of gaze effect upon neuronal responses to visual stimuli and the retinotopic visual receptive fields. This does not exclude the possibility that visual neurons with nonretinotopic fields may occur in area 7 of the parietal lobe.

\section{Discussion}

The major finding of this study is that the position of the eye in the orbit powerfully influences the responsiveness of the light-sensitive neurons of the parietal cortex. This angle of gaze effect is common, for more than $60 \%$ of the neurons studied showed this property when the animals were in the behavioral state we define as that of attentive fixation. The effect is a powerful one: the activity of an average light-sensitive neuron influenced by the angle of gaze was increased more than 3 times by a shift of gaze of $20 \%$ in the direction optimal for the effect.

The angle of gaze effect depended upon the behavioral 


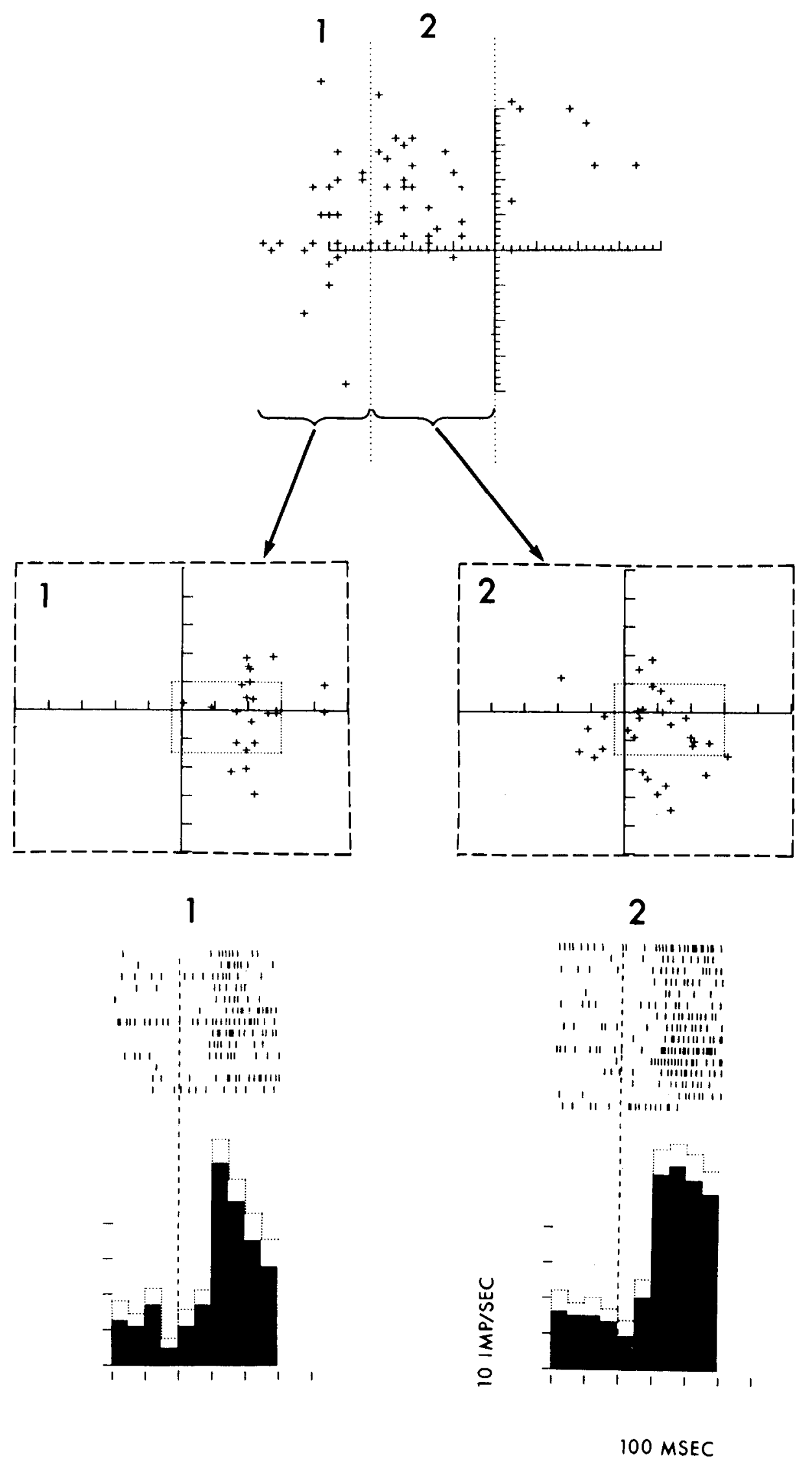

Figure 7. The upper plot shows the positions in screen coordinates of the eye pauses in the no-task state which passed stability criteria; the eyes were required to have remained stable for $200 \mathrm{msec}$ before, during, and $200 \mathrm{msec}$ after a stimulus of 100 -msec duration, making no single saccade larger than $2^{\circ}$, nor any series of smaller saccades summing to more than $5^{\circ}$. The plot above is divided into three spatial sectors by the vertical dotted lines; coordinate scale marks are $1^{\circ}$ and $5^{\circ}$. The retinal positions of the $6^{\circ} \times 6^{\circ}$ test stimuli delivered during fixations into zones 1 and 2 above are plotted on the two middle graphs. The 0,0 points on the two middle graphs now represent the points of fixation, the crosses the retinal positions of the stimuli; coordinate scale marks 
state of the animal. When the animal attentively fixated a small target and performed a detection task, the angle of gaze effect was 6 times more likely to occur than when the animal sat, quiet but alert, performing no task and glancing hither and yon about his environment.

We found no hemispheric laterality preference; the responses of neurons with smoothly graded gaze-light functions (about one-half the sample), in any one hemisphere, were nearly equally likely to be increased by ipsilateral as by contralateral shifts in the angle of gaze, and similarly as regards upward and downward shifts. The remaining half of our population of neurons were either most or least sensitive during gazes directly ahead. We do not conclude, however, that no laterality effect exists-first, because our sample of neurons studied is small; second, because we have undoubtedly lumped together in each hemisphere neurons with efferent axons and others with axons intrinsic to the cortex, many of which may be inhibitory interneurons. Such a mixture would tend to obscure any laterality effect and produce the results we obtained.

Control experiments. We interpret our results as evidence that the position of the eye in the orbit influences the excitability of the system linking the retinae to the cortex of area $7-a$. One alternative interpretation is that the charges in excitability are due to a sensory-sensory interaction between the stimuli and the slightly different, though faint, contours visible in our test chamber at different angles of gaze. The imaging of those different contours on different sectors of the retina at different angles of gaze could affect the excitability of the system by stimulating different portions of the receptive fields of neurons or by a remote, extrareceptive field effect. We believe this interpretation can be rejected, for we observed the angle of gaze effect to be present when tested in total darkness, when the only contours in the visual fields were those of the testing stimuli.

A second alternative to our interpretation is that the test stimuli delivered at different angles of gaze fell upon slightly different retinal positions, due to errors in fixation position, errors in the accuracy of positioning the testing stimuli, or to torsions of the eye at deviated gaze positions. We estimate all of these errors to be less than $1^{\circ}$, which contrasts with the large size of the receptive fields of parietal light-sensitive neurons. This, together with the fact that the retinal locations chosen for testing were at or close to the centers of those large receptive fields, suggests that these errors cannot account for the large changes in response observed.

A third alternative to our interpretation is that the stimuli were of different intensities when delivered at different positions on the tangent screen and that these intensity differences account for the differences in re- sponse observed. We believe that our observations cannot be accounted for by differences in stimulus intensity for three reasons. First, when we have varied stimulus intensity intentionally over a third of a log unit from the intensity of test stimuli-thus mimicking the differences in screen transmittance at the different positions testedwe observed no reduction in the responses. This indicates that our testing stimuli saturated the intensity functions of the parietal light-sensitive neurons. Second, if intensity changes were indeed the cause of the phenomenon observed, all neurons should have had optimal responses with gaze angles that allowed presentation of the testing stimuli near the center of the screen, where its transmittance is maximal. This is not the case, as shown by the functions of Figure 5. Third, we observed for a large number of cells that when the testing stimuli whose responses were to be compared fell at the same screen eccentricities, and thus were of identical intensities, they produced quite different responses depending upon the angle of gaze (see Fig. 11).

A fourth alternative to our interpretation is that it is the fixation distance, or the relative depth of the stimulus compared to the depth of the point of fixation, that controls the intensity of responses of parietal light-sensitive neurons. Indeed, Sakata and his colleagues (1979, 1980) have observed that these factors do indeed influence the activity of neurons of both the fixation and light-sensitive classes of parietal cells. However, the differences in the distance from the eye or the fixation points and the testing stimuli at different eccentricities on our tangent screen are small compared to those differences in fixation and stimulus distances observed by Sakata et al. to influence the responses of parietal neurons. Moreover, the results of experiments such as that of Figure 11 show remarkably strong effects of the angle of gaze upon neuronal responses under circumstances in which the stimuli concerned were delivered at the same distance as the fixation target and at identical intensities.

Therefore, we conclude that the most likely interpretation of our results is that the responses of light-sensitive neurons of the parietal cortex are strongly influenced by the position of the eye in the orbit.

What is the mechanism of the angle of gaze effect? If changes in the orbital position of the eye produce changes in the responses of parietal neurons to visual stimuli, one obvious candidate explanation of the phenomenon is that the excitability of the retino-parietal system is influenced by changes in input to the central nervous system from nonretinal orbital afferents. This appears altogether unlikely, for we rarely observed angle of gaze effects in the alert but inattentive state, even though the excitability of parietal visual neurons was then tested at a large number of angles of gaze, including those at which pow-

$=10^{\circ}$. The inner boxes of the two middle graphs enclose stimulus positions falling within the same retinal areas for the two populations of responses, evoked at different angles of gaze. The two sets of responses are illustrated by the response replicas and histograms below; stimuli began at the vertical dotted lines; histogram scales and conventions are as for Figure 1 . They show that there is no significant difference in the responses evoked during fixations into different sectors of the field of gaze in the no-task state. The outer boxes of the two middle graphs show the minimal extent of this receptive field determined in the task state; the field extended beyond the edges of our test area $\left(100^{\circ} \times 100^{\circ}\right)$. The neuron was chosen for this study because it showed a significant response in the no-task state, even though that response was greatly reduced from those evoked in the task state. 


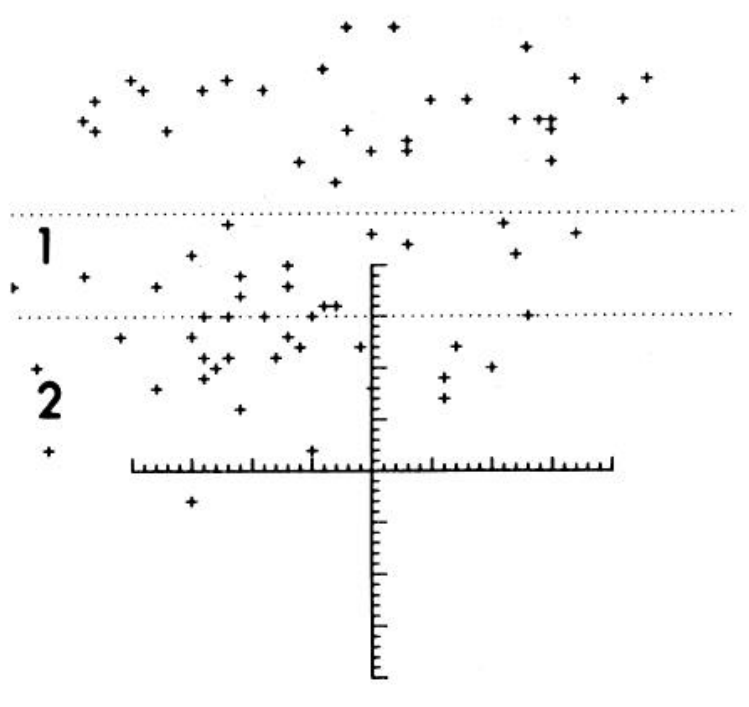

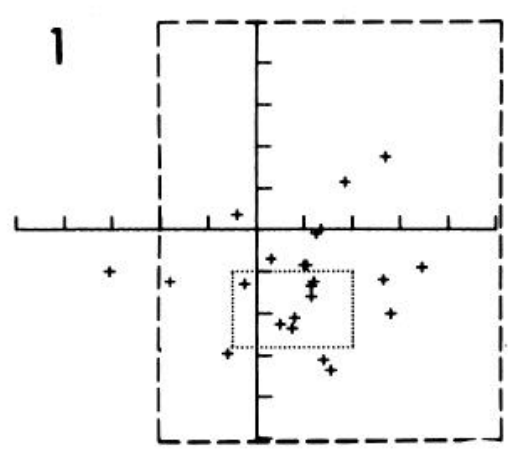

1

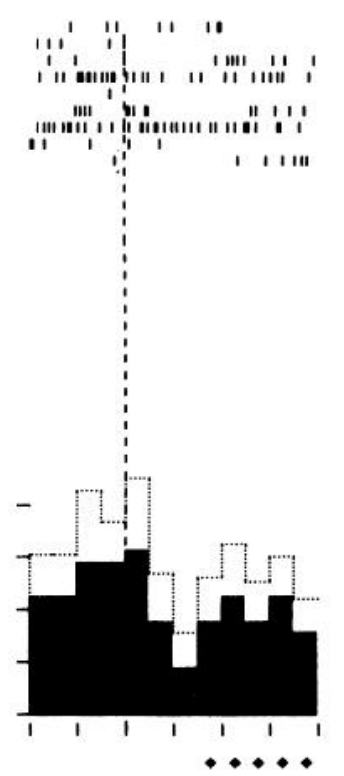

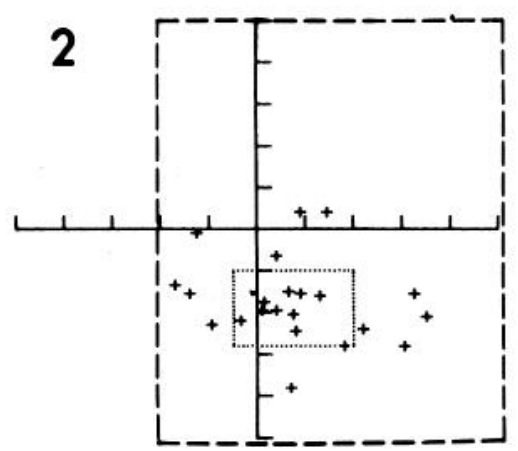

2

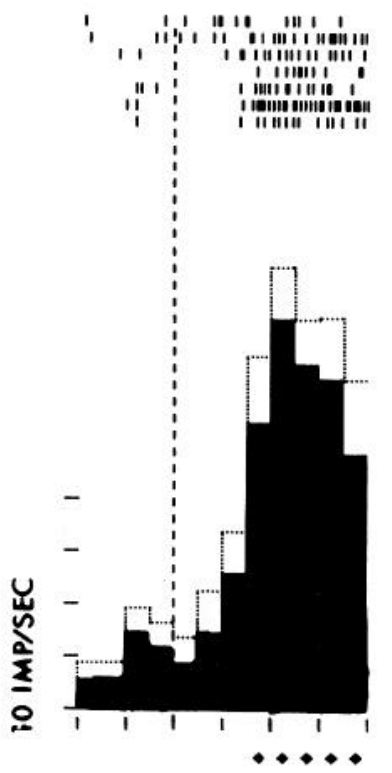

100 MSEC

Figure 8. Illustration of the results of an experiment exactly similar to that of Figure 7, except that in this case the zones of the field of gaze were divided by horizontal rather than by vertical lines. This was one of only five neurons for which we observed an angle of gaze effect upon the responses to visual stimuli delivered in the no-task state, as illustrated by the histograms below. Histogram scales and conventions are as for Figure 1. 

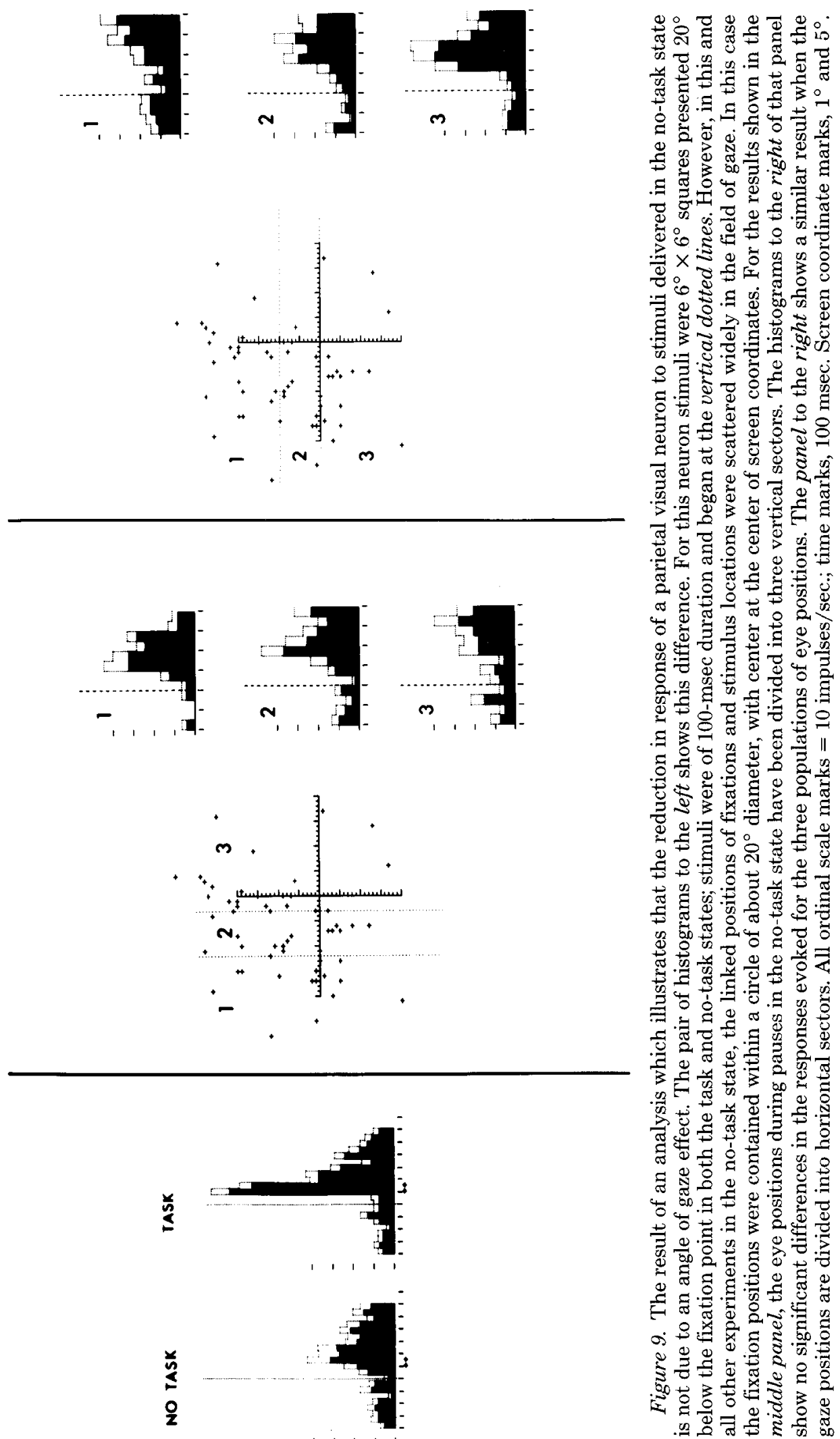

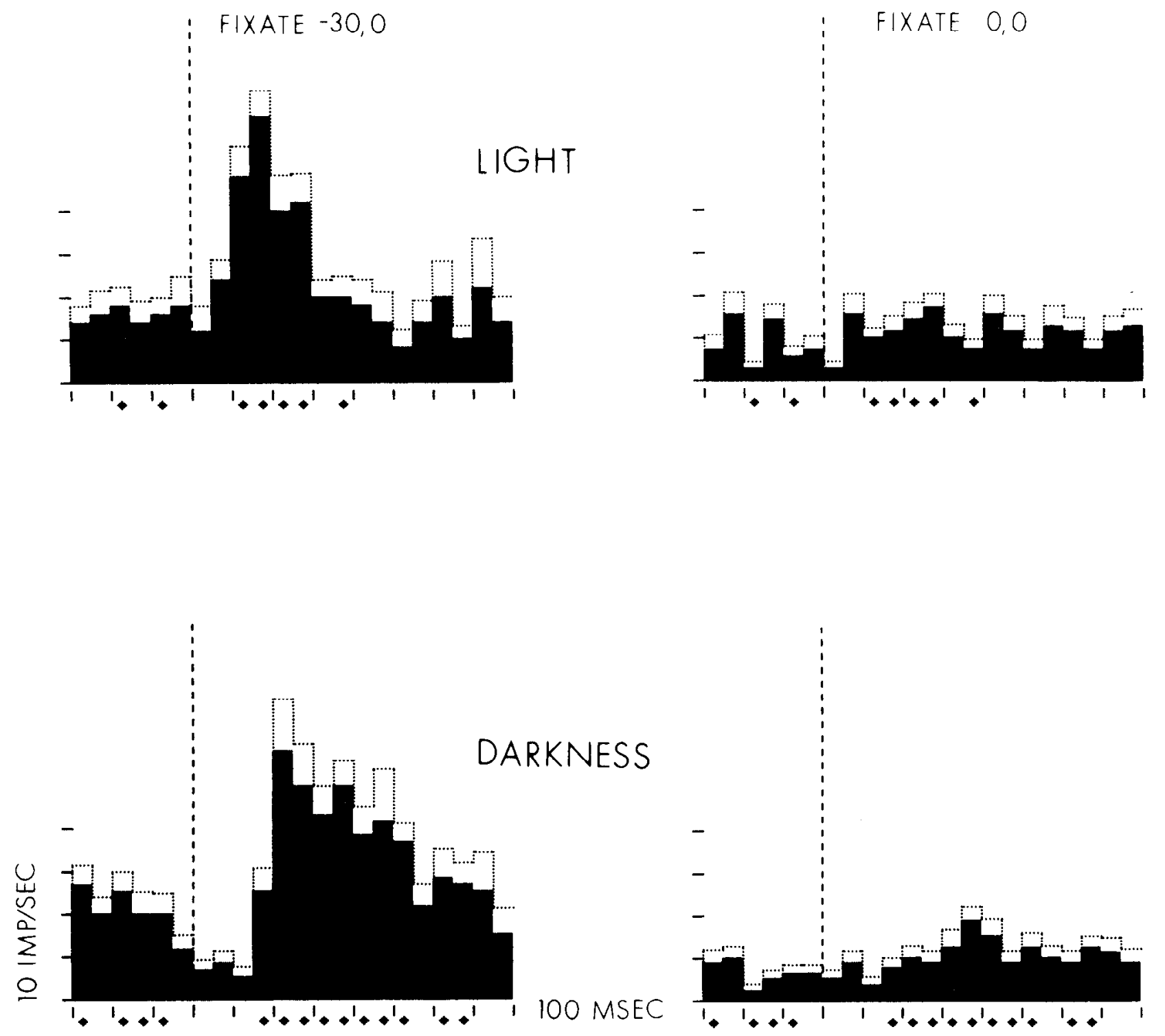

Figure 10. Darkness control. The upper pair of histograms shows a strong facilitation of the responses of a parietal visual neuron when the angle of gaze was deviated $30^{\circ}$ to the left along the horizontal meridian (upper left), as compared to the responses evoked during fixations dead ahead (upper right). In each case the stimulus was a $6^{\circ} \times 6^{\circ}$ square that began $20^{\circ}$ below the fixation point and swept upward through that point at $50^{\circ} / \mathrm{sec}$, to terminate $20^{\circ}$ above the fixation point. Stimuli began at the vertical dotted lines. Ambient light level was about $1 \mathrm{Cd} / \mathrm{m}^{2}$; stimulus intensity was about 1 log unit above ambient level. The two histograms below show the virtually identical result obtained when the experiment was repeated in darkness. Histogram conventions are as for Figure 1. Time marks, $100 \mathrm{msec}$.

Figure 11. Result of an experiment designed to test for an angle of gaze effect when depths of focus and stimulus intensities are identical. For each trio of stimuli and responses illustrated, the three test stimuli were delivered at $20^{\circ}$ intervals along the vertical meridian. The middle stimulus was located $20^{\circ}$ above the center of the screen (above), at the center (middle), or $20^{\circ}$ below the center (lower). In each case the fixation target was presented at the center of the middle stimulus of the three. Thus, the intensities of stimuli $B$ delivered $20^{\circ}$ above and $20^{\circ}$ below the center of the screen were identical, and each was reduced by about one-third log unit from the intensity of that same stimulus delivered at the center of the screen. The depth of fixation upon the central target and thus upon stimuli $B$ was identical for the upper and lower conditions illustrated. The response replicas to the right show a strong facilitation of the response of this neuron during deviations of the angle of gaze upward by $20^{\circ}$, as compared to those evoked during fixations downward by $20^{\circ}$. For the response replicas, each horizontal line indicates the period of one behavioral trial, each short upstroke an impulse discharge by the neuron. Each step deviation of a trial line indicates a behavioral event; in sequence, fixation target on, closure of the signal key by the animal (labeled $K D$, at which time all responses are synchronized), dimming of the fixation light, and release of the signal key by the animal, indicating detection. The 1-sec period of stimulus delivery is marked by horizontal lines. The nine different classes of stimuli were delivered in a randomized spatial and temporal order; the responses were later grouped by classes as shown to the right. 


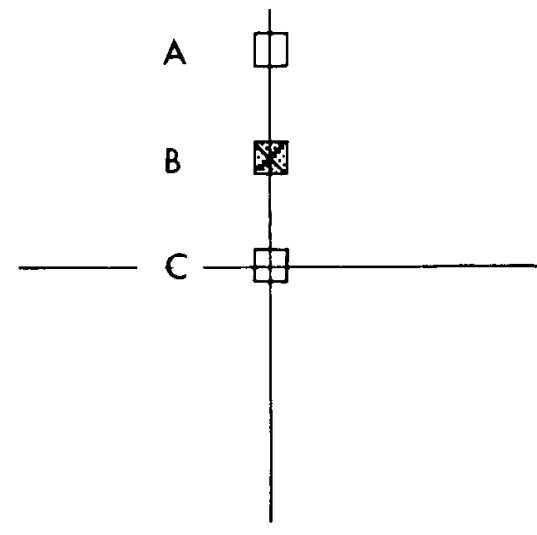

A

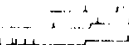

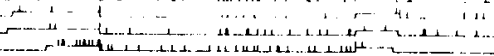

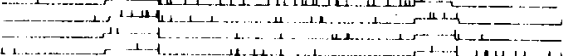

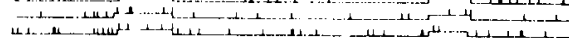

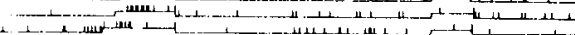

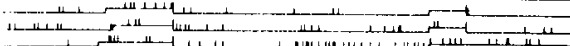

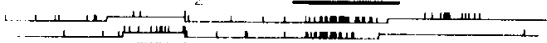

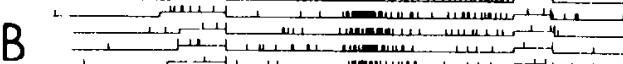

B

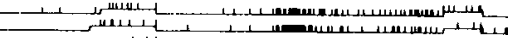

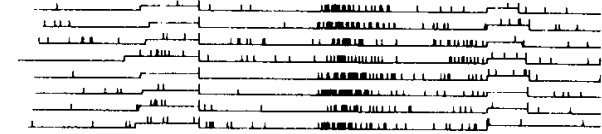

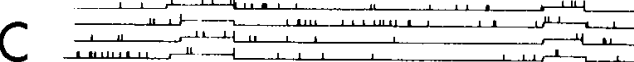

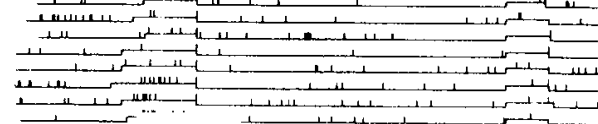

KD
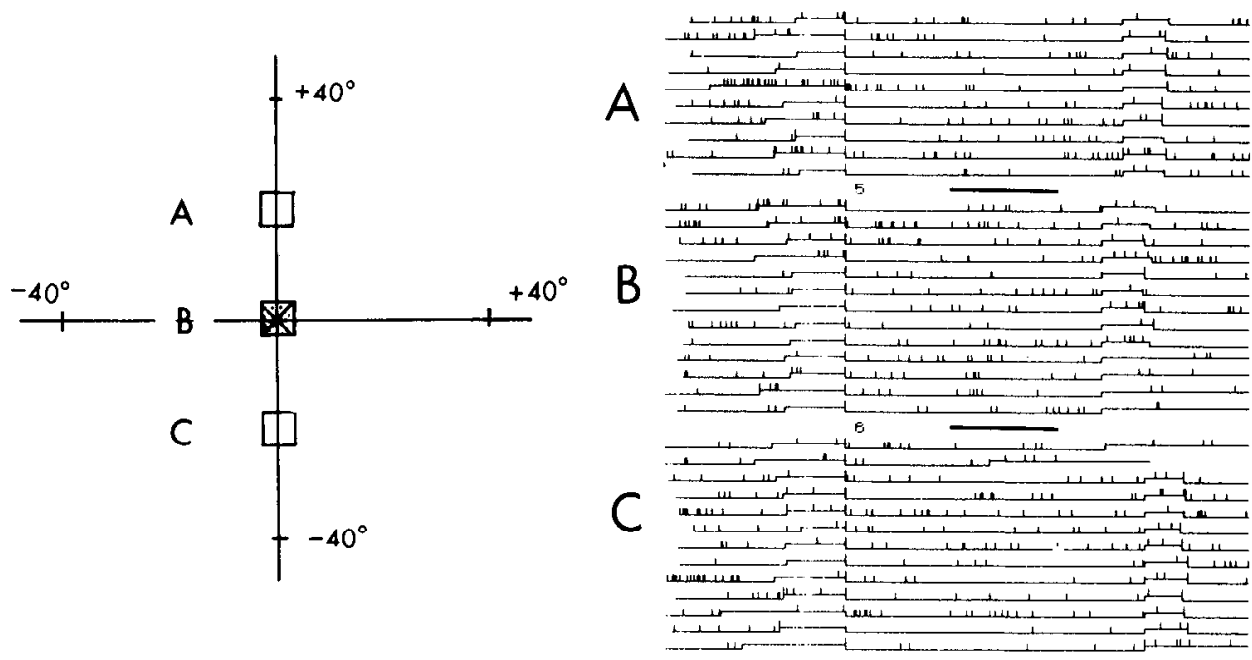

KD
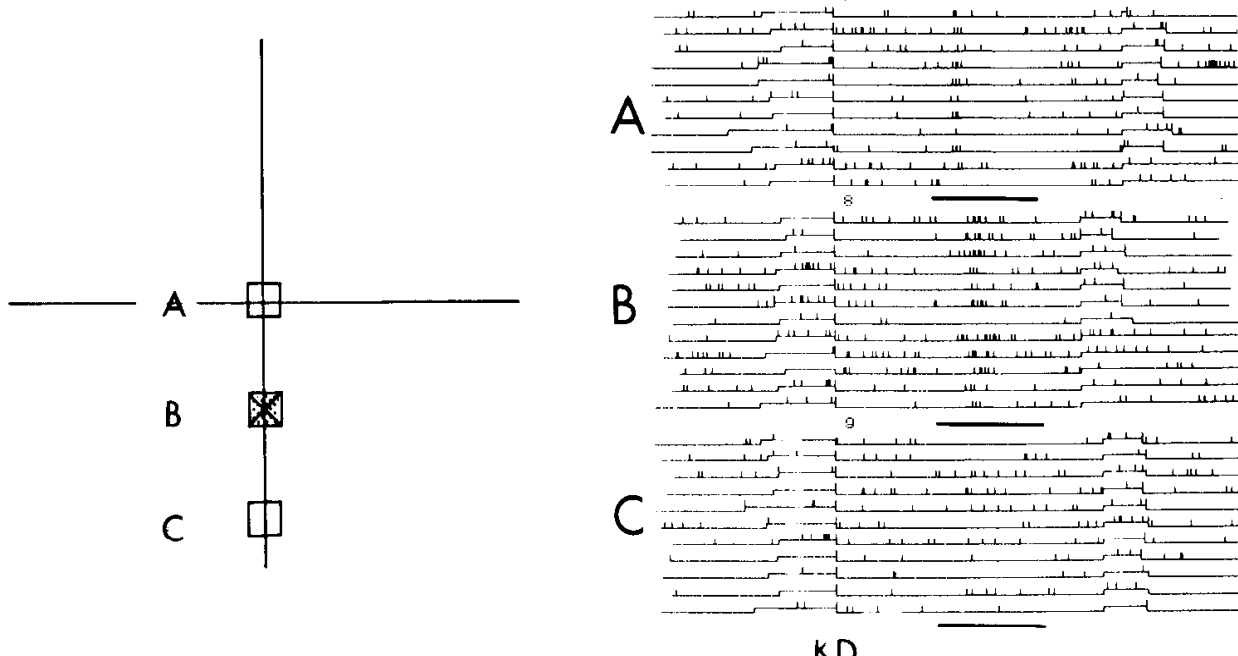

KD

Figure 11 
erful effects were observed in the behavioral state of attentive fixation. We favor the hypothesis that a reentrant signal derived from the central command system for the direction of gaze converges upon and controls the excitability of the retino-parietal pathways, perhaps in the parietal cortex itself. We have no direct evidence concerning this putative re-entrant control mechanism, although pathways linking the collicular and frontal cortical eye control mechanisms with area 7-a are now well known (for reviews see Lynch, 1980; Motter and Mountcastle, 1981). Nor can we exclude the possibility that the effect of attentive fixation is exerted earlier in the eye position control system, and thus be thought to influence the central effect of nonretinal orbital afferents.

The tight dependency of the angle of gaze effect upon the state of attentive fixation suggests that the conditioning of the responses of parietal visual neurons by the angle of gaze plays an important role in the parietal alerting mechanisms. We have previously presented some evidence (Motter and Mountcastle, 1981) that the parietal visual system has properties that suit it to function as part of the "ambient" visual system (Trevarthen, 1968), providing alerting signals that are accentuated during foveal visual attention and foveal work. The angle of gaze effect could then function to shape the spatial field of responsiveness of the ambient visual system as a condition of the line of gaze. Our own observations are so far too limited to lend any direct support to this idea of a spatial shaping function; indeed, our experiment would scarcely have revealed it if it exists. What is required is a complete mapping of the receptive fields and measures of the dynamic response properties of parietal visual neurons (such as directionality) at different angles of gaze in the different behavioral states of directed visual attention and its absence. This formidable experimental task remains for execution.

\section{References}

Andersen, R. A., and V. B. Mountcastle (1980) The direction of gaze influences the response of many light sensitive neurons of the inferior parietal lobule in the waking monkey. Soc. Neurosci. Abstr. 6: 673, 1980.

Lynch, J. C. (1980) The functional organization, of posterior parietal association cortex. Behav. Brain Sci. 3: 485-534.

Motter, B. C., and V. B. Mountcastle (1981) The functional properties of the light--sensitive neurons of the posterior parietal cortex studied in waking monkeys: Foveal sparing and opponent vector organization. J. Neurosci. 1: 3-26.

Mountcastle, V. B., R. A. Anderson, and B. C. Motter (1981) The influence of attentive fixation upon the excitability of the light-sensitive neurons of the posterior parietal cortex. J. Neurosci. 1: 1218-1235.

Sakata, H., H. Shibutani, and K. Kawano (1979) Depth seleclivilies of some visual neurons in the posterior parielal association area of the monkey. In The Integrative Control Functions of the Brain, Vol. II, pp. 83-85, Elsevier-North Holland Publishing Co., Amsterdam.

Sakata, H., H. Shibutani, and K. Kawano (1980) Spatial properties of visual fixation neurons in posterior parietal association cortex of the monkey. J. Neurophysiol. 43: 1654-1672.

Trevarthen, C. B. (1968) Two mechanisms of vision in primates. Psychol. Forschung 31: 299-337. 